\title{
Pneumoperitoneum due to pneumothorax in blunt thoracoabdominal trauma: A diagnostic challenge
}

\author{
Luis Miguel Castro* $^{\circledR}$, Rui Manuel Mendes, Coelho Fátima Borges, Capella Vanessa, Ávila Leonor \\ General Surgery Department, Hospital de Egas Moniz, Lisbon, Portugal
}

\author{
Received: 4 October 2021 \\ Accepted: 24 November 2021 \\ Published online: 5 December 2021 \\ *Corresponding author: Luis \\ Miguel Castro, Address: Avenida D. \\ José I n¹2 1A, 2720-180 Amadora, \\ Portugal. Tel: + 351915221502 , \\ E-mail: luiscastro.fmup@gmail.com \\ Competing interests: None. \\ Funding information: None. \\ Citation: Castro LM, Mendes \\ RM, Borges CF, Vanessa C, \\ Leonor A. Pneumoperitoneum \\ due to pneumothorax in blunt \\ thoracoabdominal trauma: A \\ diagnostic challenge .Journal of \\ Emergency Practice and Trauma \\ 2022; 8(1): 80-82. doi: 10.34172/ \\ jept.2021.30
}

\begin{abstract}
Objective: A Perforation of hollow viscus is the most common cause of pneumoperitoneum after a blunt thoracoabdominal trauma and demands prompt surgical exploration. Alternative routes into the peritoneal cavity, such as the presence of a diaphragmatic laceration associated with pneumothorax, although rare, should be considered when approaching these patients.

Case Presentation: We present the case of a 78-year-old male admitted to the emergency department after being ran over by a car resulting in right thoracoabdominal trauma, presenting with dyspnea and signs of peritoneal irritation. CT scan identified right pneumothorax, pneumoperitoneum and free abdominal fluid. The pneumothorax was drained and posteriorly he underwent exploratory laparotomy where a traumatic laceration of the diaphragm was identified as the cause of pneumoperitoneum.

Conclusion: Alternative causes of pneumoperitoneum should be considered in blunt thoracoabdominal trauma with possibility of conservative management in the absence of peritoneal irritation signs. Pneumothorax drainage is mandatory before intubation to avoid creation of a tension pneumothorax.

Keywords: Blunt Injuries, Traumatic Diaphragmatic Hernia, Emergency Care, Pneumoperitoneum
\end{abstract}

\section{Introduction}

Thepresence of pneumoperitoneumafterthoracoabdominal trauma is usually caused by perforation of hollow viscus, requiring surgical exploration for sepsis control (1). Rare causes of pneumoperitoneum have been described, as the air can reach the abdominal cavity from different sources through alternative routes (such as diaphragm, genitourinary tract, abdominal wall or retroperitoneum) $(2,3)$. The characteristics of the trauma forces applied and the imaging results can raise suspicion for those alternative causes of pneumoperitoneum (4). We present a case of pneumoperitoneum after traumatic pneumothorax with diaphragmatic laceration.

\section{Case Presentation}

A 78-year-old male was admitted in the emergency room after being hit by a car on his right side with complaints of dyspnea and abdominal pain. On arrival, he presented a respiratory rate of $26 \mathrm{bpm}$, muffled breath sounds on the right hemithorax, extensive right-sided subcutaneous thoracoabdominal emphysema, tachycardia (120 bpm), blood pressure of 148:70 $\mathrm{mm} \mathrm{Hg}$, a Glasgow Coma Scale of 14 and diffuse signs of peritoneal irritation on physical examination. Extended Focused Assessment with Sonography for Trauma (e-FAST) revealed a small amount of free-fluid in the right subphrenic recess and in the computed tomography (CT) scan moderate right-sided pneumothorax (Figure 1), extensive pneumoperitoneum and fat-stratification near the sigmoid colon (Figure 2). After the pneumothorax was drained, the patient was taken to the operating room for exploratory laparotomy. Intra-operative findings included a diaphragmatic laceration of $5 \mathrm{~cm}$ (Figure 3), a non-expansive haematoma of the sigmoid mesocolon and the absence of hollow viscus perforation after thorough abdominal exploration. The diaphragmatic defect was closed with non-absorbable continuous suture reinforced with non-absorbable ePTFE (expanded polytetrafluoroethylene) mesh overlapping the defect. No bowel resection was done after signs of tissue ischemia on the sigmoid colon where excluded. On the third postoperative day, a small amount of right subphrenic fluid and right pleural effusion were identified without signs of infection. No other adverse events or surgical complications were identified and the patient was discharged on the sixth postoperative day.

\section{Discussion}

High intensity blunt trauma is associated with a myriad of internal lesions that should be interpreted as a whole clinical picture, alongside with the characteristics of the 


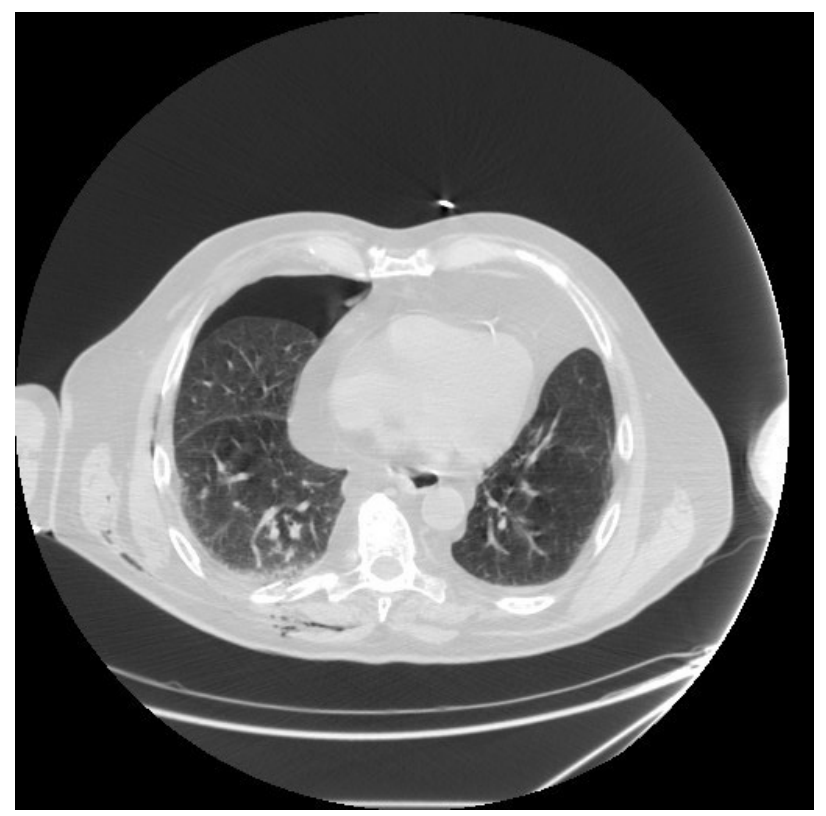

Figure 1. Computed tomography scan section of the lower thorax showing pneumothorax, rib fracture and subcutaneous emphysema

forces involved. The identification of pneumoperitoneum after a blunt thoracoabdominal trauma is associated with perforation of hollow viscus in the vast majority of cases, however the air may come from different parts of the body following a pressure gradient to the abdominal cavity (1-3).

After blunt thoracic trauma, pneumothorax is a relatively common finding with high intensity forces, generally associated with rib fractures. Usually a positive pressure gradient of 7 to $20 \mathrm{~cm}$ of water exists between the peritoneal and pleural spaces but the presence of pneumothorax increases the pleural space pressure. Also, Grosfeld et al found that when a pressure of $60 \mathrm{cmH} 2 \mathrm{O}$ in the pleural space is achieved, subcutaneous emphysema and pneumoperitoneum can occur. The presence of a diaphragmatic laceration enables the flow of air to the peritoneal space in lower pressures, further aiding the association of pneumothorax and pneumoperitoneum $(5,6)$.

Traditionally, the identification of pneumoperitoneum in a trauma patient demands prompt surgical exploration, but several studies have identified a low specificity for hollow viscus perforation in CT scan detected pneumoperitoneum, allowing for a more conservative management of some selected patients, such as a haemodynamically stable patient, with no peritoneal irritation findings and low volume pneumoperitoneum without other abdominal findings suggestive of perforation or additional lesions. The associated presence of clinical signs of peritoneal irritation significantly increases the specificity of CT scan detected pneumoperitoneum for hollow viscus perforation $(4,7)$.

Identification and drainage of a pneumothorax before tracheal intubation is of utmost importance to avoid the development of a tension pneumothorax once positive pressure is created and mechanical ventilation is initiated.

The diagnosis of an unusual origin for the pneumoperitoneum should only be made after thorough exploration of the abdominal cavity and exclusion of more common aetiologies (such as bowel perforation) and other associated gastrointestinal lesions are taken into account.

The reported overall incidence of diaphragmatic hernias after blunt thoraco-abdominal trauma is between $1 \%$ and $20 \%$ with a great percentage of these unidentified in the inciting episode. Left-sided hernias are more common possibly due to the protective effect of the area covered by the liver on the right side and more fibrous tissue on the

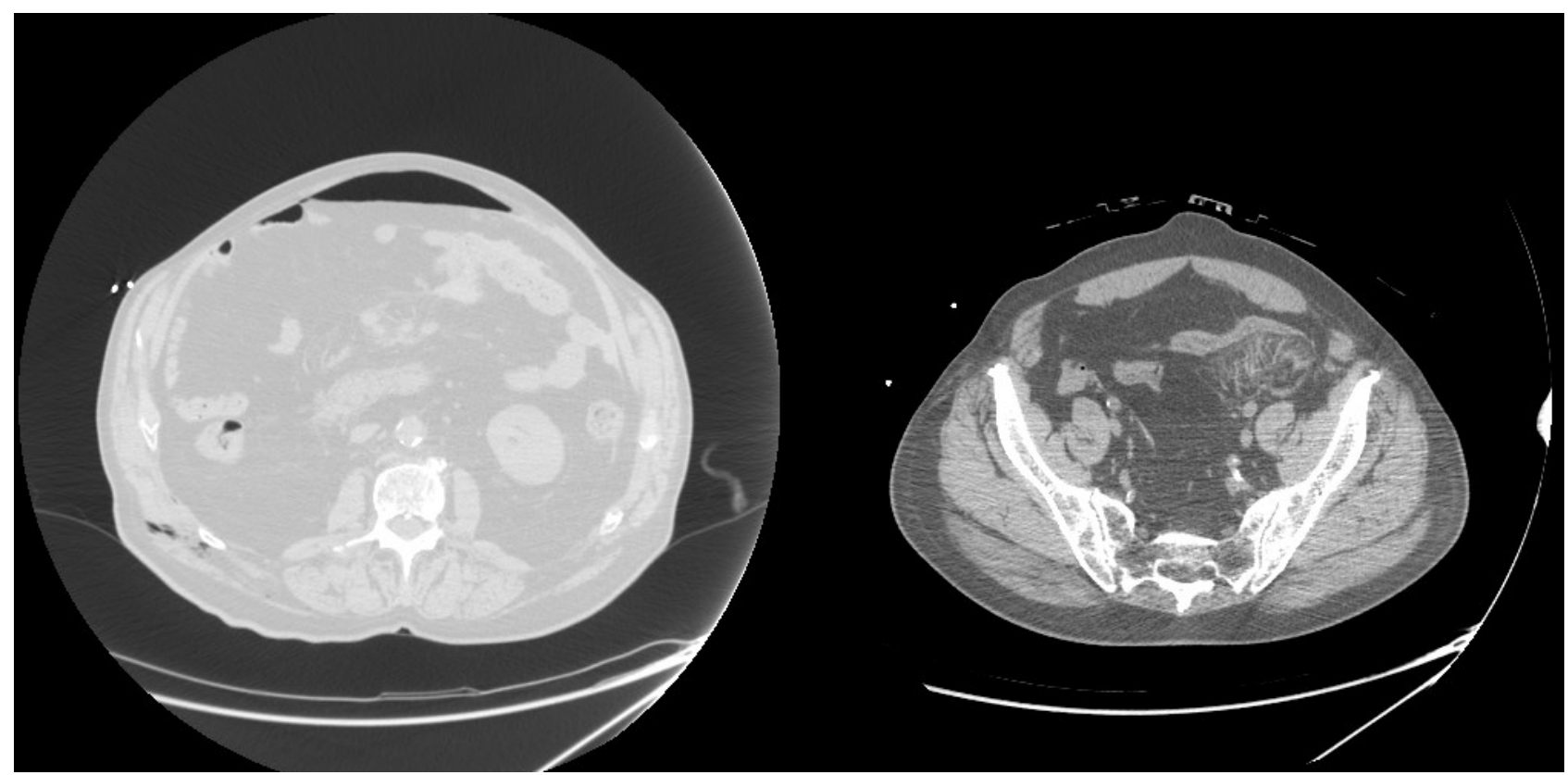

Figure 2. Computed tomography scan sections of the abdomen with pneumoperitoneum, subcutaneous emphysema and fat stratification near the sigmoid colon. 


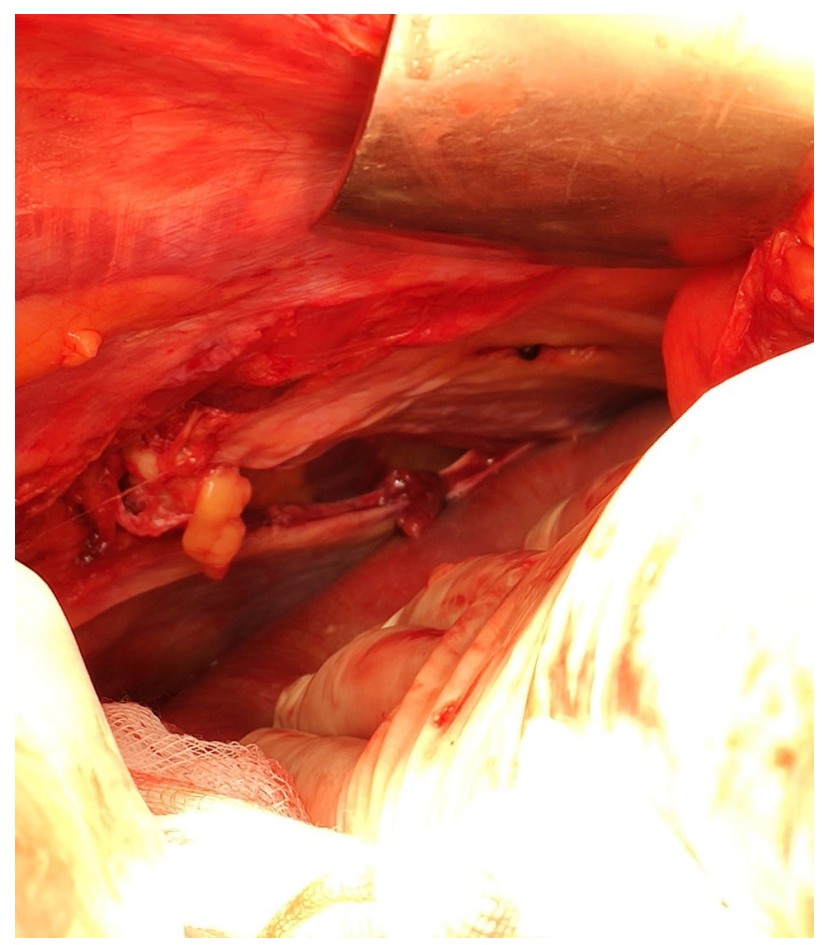

Figure 3. Intra-operatory finding of diaphragmatic traumatic laceration on the right side.

left. Recently, the common use of preoperative CT scans decreased the number of undiagnosed diaphragmatic hernia (DH's). Repair of DH's should be done once the defect is recognized, if possible in the inciting event, avoiding late complications of strangulation. Primary repair is possible if the defect is small and no tension on the repaired tissue is present. In case of a large defect, severe tissue damage or primary repair under tension, mesh repair is recommended to reduce recurrence, but no consensus on mesh type is found in the literature. In cases of contaminated field, such as concomitant bowel perforation, use of biological mesh is an option to reduce infection rate (8).

In the case described, the presence of pneumothorax, several rib fractures and subcutaneous emphysema could raise the suspicion for an alternative origin for intra-peritoneal free air. But peritoneal irritation signs were present and CT scan showed alterations of the mesosigmoid, consequently, urgent surgical exploration was performed. The diaphragmatic defect was corrected with mesh placement due to concern on tissue tension after simple closure.

\section{Conclusion}

Pneumoperitoneum after blunt abdominal or thoracoabdominal trauma is not always due to hollow viscus perforation and other sources of free intraperitoneal air should be considered, particularly in high intensity settings.

Careful selection of patients to undergo conservative management can be useful to avoid the high morbidity and mortality of an urgent surgical exploration. However, the presence of peritoneal irritation signs, haemodynamic instability or suspected multiple injuries warrants surgical exploration either by a laparoscopic or an open approach. Thoracic drainage is mandatory in every case of pneumothorax before starting invasive ventilation to avoid creating a tension pneumothorax.

\section{Authors' contributions}

RMM, CFB and CV: Surgical Team, Scientific review

AL: Scientific review. All authors made substantial contribution to the conception and design of the work and to the drafting and revision of the article content. The final version submitted as the approval of all authors which are accountable for all aspects of the work in ensuring that questions related to the accuracy or integrity of any part of the work are appropriately investigated and resolved.

\section{Ethical issues}

Informed consent statement was obtained from the patient for the publication of this report.

\section{References}

1. American College of Surgeons Committee on Trauma. ATLS: Advanced Trauma Life Support Student Course Manual. 10th ed. Chicago: American College of Surgeons; 2018. p. 82-99.

2. Di Saverio S, Filicori F, Kawamukai K, Boaron M, Tugnoli G. Combined pneumothorax and pneumoperitoneum following blunt trauma: an insidious diagnostic and therapeutic dilemma. Postgrad Med J 2011; 87(1023): 75-8. doi: 10.1136/pgmj.2010.110262.

3. Allan Z, Peng C, Chandra R. Traumatic diaphragmatic rupture with underlying lung laceration and tension pneumoperitoneum. J Surg Case Rep 2017; 2017(6): rjx120. doi: $10.1093 /$ jscr/rjx120.

4. Marek AP, Deisler RF, Sutherland JB, Punjabi G, Portillo A, Krook J, et al. CT scan-detected pneumoperitoneum: an unreliable predictor of intra-abdominal injury in blunt trauma. Injury 2014; 45(1): 116-21. doi: 10.1016/j. injury.2013.08.017.

5. Grosfeld JL, Boger D, Clatworthy HW Jr. Hemodynamic and manometric observations in experimental airblock syndrome. J Pediatr Surg 1971; 6(3): 339-44. doi: 10.1016/0022-3468(71)90476-3.

6. Williams NM, Watkin DF. Spontaneous pneumoperitoneum and other nonsurgical causes of intraperitoneal free gas. Postgrad Med J 1997; 73(863): 531-7. doi: 10.1136/ pgmj.73.863.531.

7. Ahmad R, Mohamad N, Latiff AK, Ahmad Z, Idrus II. Pneumoperitoneum following blunt abdominal injury: does it warrant laparotomy. Int J Case Rep Imag 2011; 2(12): 23-7. doi: 10.5348/ijcri-2011-12-76-CR-6.

8. Filosso PL, Guerrera F, Sandri A, Lausi PO, Lyberis P, Bora G, et al. Surgical management of chronic diaphragmatic hernias. J Thorac Dis 2019; 11(Suppl 2): S177-S85. doi: 10.21037/jtd.2019.01.54. 